\title{
DEVELOPMENT OF MRONJ IN A FEMALE PATIENT WITH MULTIPLE MYELOMA AFTER 10 YEARS OF IMPLANTS REHABILITATION: A CASE REPORT
}

Lia Ester Argiolas, Martina Salvatorina Murgia ${ }^{1}$, Cinzia Casu, Agostino Garau, Valentino Garau ${ }^{1}$

1 University of Cagliari

Funding: The author(s) received no specific funding for this work.

Potential competing interests: The author(s) declared that no potential competing interests exist.

\section{Abstract}

Medication-related osteonecrosis of the jaw (MRONJ) is an adverse drug reaction that affects the mandible and maxilla of patients exposed to bone-targeting agents (anti-resorptive and anti-angiogenic agents). Bone lesions in multiple myeloma require bisphosphonate treatment. Particularly, the aim of this abstract is to report a successfully treated case of type $2 \mathrm{ONJ}$ in a patient with Multiple Myeloma (MM) undergone to intravenous (IV) Zolendronic Acid (ZA).

\section{Background:}

Medication-related osteonecrosis of the jaw (MRONJ) is an adverse drug reaction that affects the mandible and maxilla of patients exposed to bone-targeting agents (anti-resorptive and anti-angiogenic agents) ${ }^{[1]}$. The aim of this abstract is to report a successfully treated case of type $2 \mathrm{ONJ}$ in a patient with Multiple Myeloma (MM) undergone to intravenous (IV) Zolendronic Acid (ZA). 


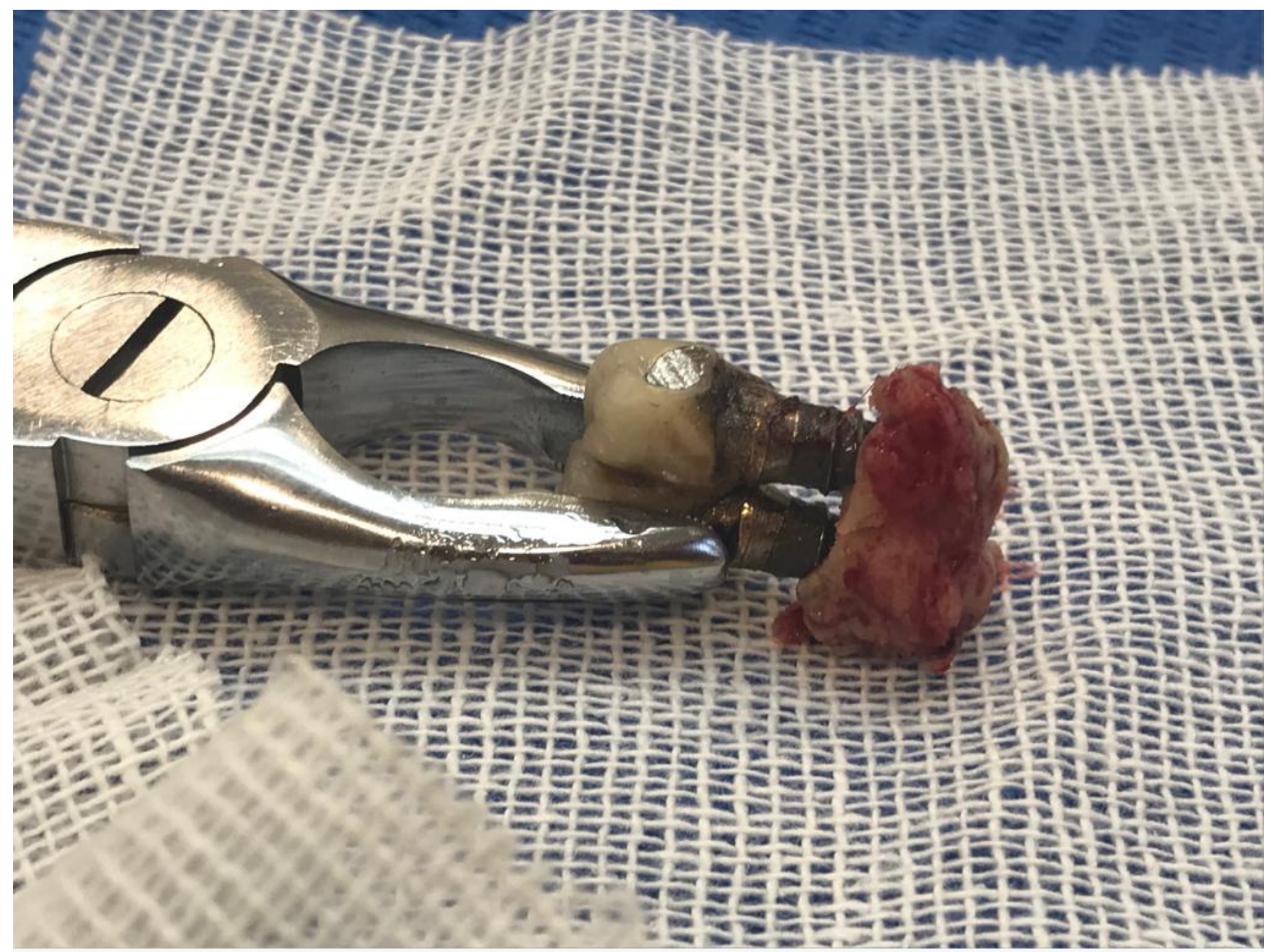

Fig. 1 Sequestrectomy with implants performed.

Materials and Methods: A 76-years-old woman came to our attention in September 2018. Her medical history showed a MM's relapse treated with IV ZA, Carfilzomib, Dexamethasone and Lenalidomide. Furthermore, the patient reported that had received dental implants about 10 years before the start of therapy with ZA. She later developed MRONJ which began in the peri-implant areas. Physical examination showed preternatural dental mobility, diffuse inflammation with persistent pain. Imaging (dental panoramic tomograph and TC dental scan) showed diffuse bone resorption. The suspected diagnosis was of a lesion compatible with MRONJ. A previous antibiotic cycle allowed a clinical improvement. Blood chemistry tests were required to evaluate liver and kidney function. Local therapy with the application of gel and mouthwash with Chlorhexidine was prescribed. Periodic clinical checks were recommended every 20-30 days. During the check-up there was an improvement in the local state: absence of spontaneous pain, but there were dental mobility, especially of element 44, and exposure of implants turns of the second and fourth quadrant. In February 2019 the presence of a purulent exudate was highlighted in the fourth quadrant associated with local swelling. Following ministerial guidelines (S.I.P.M.O.), it was decided to program the avulsion of element 44 and of the implants in seat $45-46$ under antibiotic prophylaxis. Treatment consisted of simultaneous amoxicillin and clavulanic acid (cp $3 \mathrm{~g} / \mathrm{day}$ ) and metronidazole (cp 1,5 g/day) from the day before up to six days after the surgery. During the surgical procedure, a block of necrotic bone of about $20 \mathrm{~mm}{ }^{*} 6 \mathrm{~mm}$ in diameter was removed together with the implants (Fig. 1). The surgical toilet of the site was 
performed then closed for the first purpose with a silk suture. In October 2019 it was decided to also remove the implants of the second quadrant due to the increasing mobility. The same clinical and surgical protocol was followed.

Results: In subsequent check-ups the situation is stable: absence of pain and absence of inflammation. To date, the mucous membrane appears healthy. The patient is monitored every 20 days, follows closed home oral hygiene measures (mouthwash and gel with Chlorhexidine). In literature there are few cases of peri-implant zoledronic acid-ONJ in cancer patients who underwent implant insertion before the development of malignancy ${ }^{[2]}$, including one of $\mathrm{MM}^{[3]}$. Consequently, it would appear that patients on ZA therapy have a higher risk of developing peri-implant MRONJ, although more cases and clinical trials are needed to delineate the risk profile.

\section{Conclusions:}

MRONJ can arise in peri-implant bone, especially when the implants were placed long before the development of malignancy (and consequent intake of related drugs). For this reason, it's advised that patients who are or have been treated with anti-resorptive or anti-angiogenic agents and who also have dental implants, should undergone closer checks in order to prevent the possible appearance of ONJ.

\section{References}

1. 1. Mauceri R, Panzarella V., Morreale I., Campisi G. Medication-related osteonecrosis of the jaw in a cancer patient receiving lenvatinib. J Oral Maxillofac Surg. 2019;48(12):1530-1532.

2. Marín-Fernández A., Medina B., Aguilar-Salvatierra A., Jiménez-Burkhardt A., Moreno G. Jaw osteonecrosis management around a dental implant inserted 2 years before starting treatment with zoledronic acid. G. J Clin Exp Dent. 2015; 7(3): e444-e446.

3. Junquera L., Gallego L., Pelaz A. Multiple Myeloma and Bisphosphonate-Related Osteonecrosis of the Mandible Associated with Dental Implants. Case Rep Dent. 2011; 568246. 